\title{
A Study of Forward Error Correction Schemes for Reliable Transport in Underwater Sensor Networks
}

\author{
${\text { Bin } \operatorname{Liu}^{\dagger} \text {, Florent Garcin }}^{\ddagger}$, Fengyuan Ren ${ }^{\dagger}$ and Chuang $\operatorname{Lin}^{\dagger}$ \\ $\dagger$ Department of Computer Science and Technology \\ Tsinghua University, Beijing, China \\ $\ddagger$ School of Computer and Communication Sciences \\ Swiss Federal Institute of Technology (EPFL), Lausanne, Switzerland
}

\begin{abstract}
Underwater communications is a very challenging topic due to its singular channel characteristics. Most protocols used in terrestrial wireless communications can not be directly applied in the underwater world. A high bit error rate and low propagation delay make the design of reliable transport protocols especially awkward.

In this paper $^{1}$, we first propose four schemes that combine forward error correction mechanisms at the bit and/or packet level to increase the reliability in a non-cooperative scenario. The broadcast property of the underwater environment allows us to extend them to a cooperative setting. Based on our analyses, we introduce ADELIN: an ADaptive rELIable traNsport protocol for underwater sensor networks. We suggest an architecture for implementation and compare our protocol to other schemes. We show that it succeeds in a better probability and energy tradeoff for both single- and multi-hop communications.
\end{abstract}

\section{INTRODUCTION}

Understanding the key mechanisms of the oceans is crucial for the knowledge of our Earth's climate and atmosphere. Although water covers most of our planet, the underwater world remains hostile to humans and little explored. Over the past few years, there has been a relentless effort to investigate the abyssal depths of the oceans. This infatuation is highly motivated by the applications of underwater communication, such as undersea resources exploration and exploitation, and their economic impacts.

Indeed, underwater sensor networks (UWSNs) have many applications in military and civilian environment. The former includes for instance monitoring or surveillance. Fortunately, UWSNs can be used in a smarter and more useful way, for example in oceanographic data collection, environmental monitoring or disaster prevention.

Communications in underwater is a very challenging topic to which researchers have shown only recently a growing interest [1], [2]. Since radio waves do not propagate well in this environment, communication is done by the mean of acoustic channel and differs in many aspects.

Compared to terrestrial wireless communication, the propagation speed is very slow (around $1.5 \cdot 10^{3} \mathrm{~m} / \mathrm{s}$ ). It varies with the pressure (depth), temperature and salinity and thus highly depends on the environment. The path loss is caused by spreading and absorption and is related not only to the distance between nodes but also to the frequency. This implies that

\footnotetext{
${ }^{1}$ First two authors equally contributed to this work
}

underwater acoustic communications have limited bandwidths [3]. In addition, the noise is not the same and comes from various sources such as the movement of water, rain and wind, seismic and volcanic activities or biological phenomena. Finally, in shallow water, the signal reflection from the surface and seabed creates multipaths. In deep water, it occurs as well for instance due to topographic sources like hills, cliffs or hollows.

Due to these interesting features, the design of reliable transport protocols is tricky. Generally, reliability is guaranteed by providing either feedback or redundancy. Feedback-based protocols like automatic repeat request (ARQ) mechanisms are not appropriate mainly because of the propagation delay but also because of the energy consumption. In densely deployed sensor networks with feedback-based protocols, there may be multiple sensor nodes in the transmission range of the sender and if the receiver requests a retransmission, other nodes will waste energy to overhear the duplicate packets and response to the feedbacks. Furthermore, in the case of lost positive feedbacks, this family of protocols not only degrade the channel usage and increase the transmission latency, but also induce the sender to retransmit packets that are already successfully received.

For these reasons and to achieve a high reliability, we propose four schemes that combine forward error correction (FEC) mechanisms at the bit and/or packet level in noncooperative scenario. At the bit level, we select the binary $\mathrm{BCH}$ code and at the packet level, erasure coding (EC). The broadcast property of the underwater environment allows us to extend them to a cooperative setting. Although these techniques might have a high probability, we should take into account their respective encoding and decoding energies.

This paper begins with a brief review of the literature on reliable transport protocol in both terrestrial and underwater environments. Then, in Section III, we define in more details the characteristics of acoustic channels and give the mathematical model we use for communications. We combine $\mathrm{BCH}$ and/or EC to introduce four schemes and we analyse them in two different scenarios: with and without cooperation (Sec. V and IV) between nodes. Based on these analyses, Section VI presents ADELIN, an adaptive reliable transport protocol, and its architecture. Simulations are conducted in Section VII and demonstrate the benefits of our proposed protocol. Finally, we 
conclude our work in Section VIII.

\section{RELATED WORK}

Reliable transport protocols for terrestrial wireless sensor networks have been studied in many publications [4], [5]. In the following, we will summarise the main contributions of several protocols for either terrestrial or underwater sensor networks [6], [7].

The idea of Pump Slowly, Fetch Quickly (PSFQ) [4] is to slowly pump data packets one after another into the network using a large period and a broadcast mechanism. Nodes receiving those packets store them into a cache and when they are received in-sequence, forward them to downstream nodes. An intermediate node receiving an out-of-sequence packet does not forward it immediately, but quickly requests the missing packet from the upstream neighbour. This operation is called fetch and corresponds to a NACK. Between two pumps, multiple fetch trials may be made. This hop-by-hop recovery is aggressive and tends to delay the delivery of the next packets. Thus, when applied to underwater sensor networks, the total delivery time drastically increases.

Reliable Multi-Segment Transport (RMST) [5] is another NACK-based reliable transport protocol aiming at delivering large data packets. It combines end-to-end and hop-by-hop retransmissions in the MAC and transport levels. Intermediate nodes use caches for fast recovery when a packet loss is detected. In the worst case, the retransmission request has to travel all the way back to the source.

PSFQ and RMST are based on ARQ mechanism and thus, not appropriate for underwater sensor networks. So far, reliable transport protocols for underwater sensor networks have not been addressed except recently in [6], [7].

In [6], the authors introduce a per-hop hybrid implicit/explicit acknowledgement scheme for Stop and Wait ARQ in a multi-hop acoustic channel. In this scheme, when a relay node receives a packet, it sends an acknowledgement message only when its previous data transmission is already acknowledged. The acknowledgement can be implicit with the data packet itself or explicit with an acknowledgement message. Unfortunately, both mechanisms have a high timeout. They demonstrate that their protocol has a better latency and energy efficiency than traditional schemes. However, the latency remains high and when explicit acknowledgement is used, it increases the energy and communication costs.

[7] proposes a Segmented Data Reliable Transport protocol called SDRT. SDRT is a hybrid of FEC and ARQ. It uses erasure codes (simple variant of Tornado codes) to send data block by block and hop by hop. Basically, the source encodes and sends a data block to the next node. The intermediate node decodes, reconstructs and encodes again the data block. Then it forwards it to the next node. The sender continues to pump encoded packets on the channel until it receives a positive acknowledgement message from its next node. SDRT reduces the total number of transmitted packets, improves channel utilisation and simplify protocol management. On the other hand, the main drawback comes from the utilisation of ARQ.
Indeed, SDRT keeps sending packets until a positive feedback which obviously wastes energy. Furthermore, if suddenly a node stops relaying, the sender will hardly detect it which leads to a huge communication cost.

\section{ChARACTERISTICS OF UNDERWATER ACOUSTIC CHANNELS}

We have previously stated that underwater communications diverge from terrestrial wireless communications. In this section, we mathematically characterise the features of underwater acoustic channels and define the model used in the rest of this paper.

Since the acoustic signal is prone to multipath propagation, we model the underwater acoustic channel as a Rayleigh fading one [8]. We also take the most commonly used binary phase shift keying (BPSK) as the standard to calculate the average bit error rate (BER).

We define $\gamma_{s}$ and $\gamma_{b}$ as the signal-to-noise ratio (SNR) per symbol and per bit respectively. In BPSK, one symbol error corresponds exactly to one bit error, i.e. $\gamma_{s}=\gamma_{b}$. Let

$$
\overline{\gamma_{s}}=10^{\gamma_{s} / 10}=10^{\gamma_{b} / 10}
$$

We can get the average BER of BPSK in an underwater Rayleigh fading channel [9]:

$$
\operatorname{BER}\left(\gamma_{b}\right)=\frac{1}{2}\left(1-\sqrt{\frac{\overline{\gamma_{s}}}{1+\bar{\gamma}_{s}}}\right)
$$

The passive sonar equation [10] describes the SNR per bit of a signal from a source at the receiver:

$$
\gamma_{b}=S L-T L-N L+D I
$$

where $S L$ is the source level, $T L$ the transmission loss, $N L$ the noise level and $D I$ the directivity index. All quantities are in $\mathrm{dB}$. In the following, we will consider omnidirectional hydrophones which imply that the diversity index is 0 .

The transmission loss over a distance $d$ in meter for a signal of frequency $f$ in $\mathrm{kHz}$ is given by

$$
T L=k \cdot 10 \log _{10} d+\alpha(f) d \cdot 10^{-3}
$$

where $k$ is the spreading factor (spherical, cylindrical, ...) and $\alpha(f)$ the absorption coefficient. In the following, we assume a cylindrical spreading factor $k=1$.

We use the Thorp's formula [10], [11] for the absorption coefficient in $\mathrm{dB} / \mathrm{km}$ :

$\alpha(f)=0.11 \frac{f^{2}}{1+f^{2}}+44 \frac{f^{2}}{4100+f^{2}}+2.75 \cdot 10^{-4} f^{2}+0.003$

with $f$ in $\mathrm{kHz}$.

The noise is composed of four components: turbulence, shipping, waves and thermal noise. The empirical formulae are given in [12]. For simplicity, we employ a useful approximation presented in [3], namely:

$$
N L=50-18 \log _{10} f
$$

$f$ is in $\mathrm{kHz}$. 


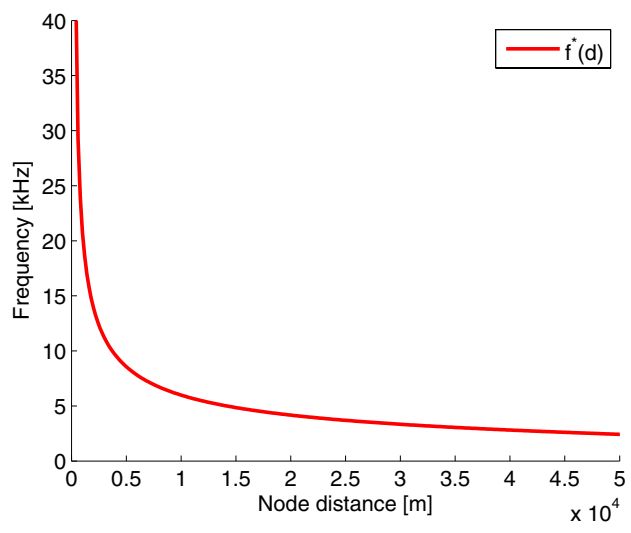

Fig. 1. Optimal frequency

From Equations 3, 4 and 6, we obtain:

$$
\gamma_{b}=S L-10 \log _{10} d-\alpha(f) d \cdot 10^{-3}-50+18 \log _{10} f
$$

We have seen in Equation 2 that the SNR per bit $\gamma_{b}$ directly determines the BER. In perspective to increase the transmission reliability, we can get its maximum value by computing the derivative $\frac{\partial \gamma_{b}}{\partial f}$ :

$\frac{\partial \gamma_{b}}{\partial f}=\frac{18}{f \ln 10}-f d\left(\frac{2.2 \cdot 10^{-4}}{\left(1+f^{2}\right)^{2}}+\frac{360.8}{\left(4100+f^{2}\right)^{2}}+5.5 \cdot 10^{-7}\right)$

In UWSNs, the node distance $d$ is usually in the range of 0.1 to $50 \mathrm{~km}$. Thus, it is obvious that $\forall d \in[0.1,50]$ :

$$
\begin{aligned}
\lim _{f \rightarrow 0} \frac{\partial \gamma_{b}}{\partial f} & =+\infty \\
\lim _{f \rightarrow+\infty} \frac{\partial \gamma_{b}}{\partial f} & =-\infty
\end{aligned}
$$

In other words, for $d \in[0.1,50]$, there exists at least one frequency where the SNR per bit is maximum. Actually, for $d \in[0.1,50]$, there is only one positive root (optimal frequency) for equation $\frac{\partial \gamma_{b}}{\partial f}=0$. This optimal frequency, denoted by $f^{*}(d)$, is plotted in Figure 1 and will be used in the rest of this paper as the transmission frequency.

The sound intensity of a source is related to a reference intensity and is given by

$$
I_{t}=10^{S L / 10} I_{\text {ref }}
$$

where $I_{\text {ref }}=\frac{p^{2}}{2 \rho c}$ with $p$ is the effective sound pressure, $\rho$ the density of sea water and $c$ the propagation velocity of the sound wave in sea water. The speed of sound varies with the pressure, temperature and salinity and thus depends on the environment. The pressure depends on the depth as well. For simplicity, we assume a constant speed of $c=1500 \mathrm{~m} / \mathrm{s}$. Thus, we take $I_{\text {ref }}=0.67 \cdot 10^{-18} \mathrm{~W} / \mathrm{m}^{2}$.

In the case of cylindrical spreading, the power $P_{t}$ required to achieve intensity $I_{t}$ at $1 \mathrm{~m}$ from the source in the direction of the receiver is expressed as

$$
P_{t}=2 \pi z I_{t}
$$

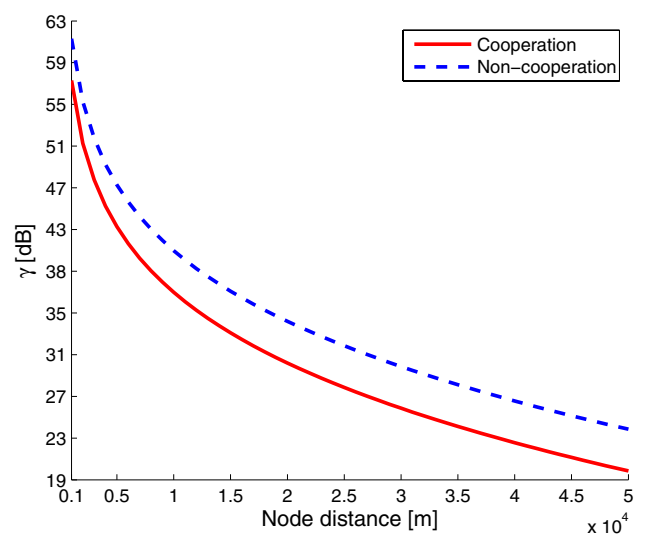

Fig. 2. Signal-to-noise ratio per bit for the two scenarios

where $P_{t}$ in watt and $z$ is the depth in meter.

In Sections IV and V, we will study two different scenarios: a simple communication between two nodes and the cooperation with the help of a third relaying node. The latter does not need to have a high source level while in the former, it is required. According to [10], the SNR at the receiver should be around 20 to $24 \mathrm{~dB}$. Consequently, we pick up two source levels $S L$ of 122 and $118 \mathrm{~dB}$, for the simple and cooperative scenarios respectively, to guarantee this SNR at a distance of $50 \mathrm{~km}$. Figure 2 illustrates Equation 7 with the optimal frequency $f^{*}(d)$.

\section{NON-COOPERATION}

We first start to study a simple scenario where a source node wants to send data to a destination over a single path. To find the appropriate FEC combination, we have selected four different implementations as depicted on Figure 3. The first and second encode the data packets with $\mathrm{BCH}$ and $\mathrm{EC}$ respectively. The third applies EC on the data packets, then $\mathrm{BCH}$ on the data packets only. The last applies EC on the data packets, then $\mathrm{BCH}$ on the data and check packets. We will refer these implementations as Scheme 1, Scheme 2, Scheme 3 and Scheme 4 respectively.

We consider the binary $\mathrm{BCH}$ code with the following property. For any $m$ and $t$, there exists a binary $\mathrm{BCH}$ code of block length $n=2^{m}-1$ and parity check (overhead bits) of at most $\phi=m t$ that can correct up to $t$ errors. For erasure coding, we take the Reed-Solomon code with the assumption that we can reconstruct $k$ original data packets by receiving any $k$ packets out of $k+s$ packets, with $s$ check packets.

\section{A. Probability}

We have seen previously that the bit error rate depends on the signal-to-noise ratio (Eq. 2). However, when we use the optimal frequency $f^{*}(d)$ in Equation 7, the distance between the sender and receiver determines the bit error rate. Thus, we use the notation $B E R(d)$ instead of $B E R\left(\gamma_{b}\right)$ in the following. 


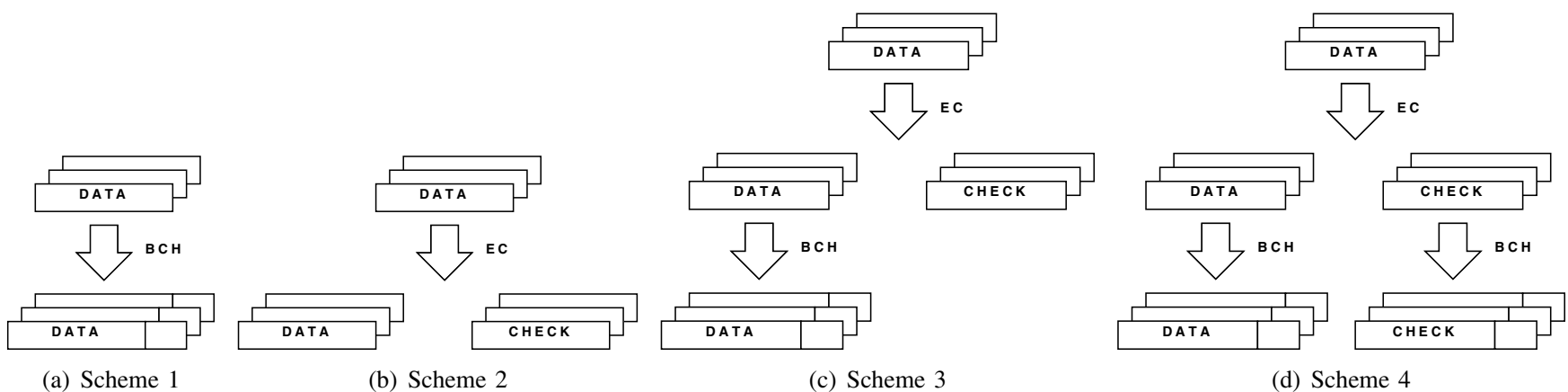

Fig. 3. Different schemes

The probability of a successful transmission for a single packet of length $l$ over one hop is

$$
p(l, d)=(1-B E R(d))^{l}
$$

and when $\mathrm{BCH}$ coding is used, we have

$$
p_{B C H}(l, t, d)=\sum_{i=0}^{t}\left(\begin{array}{l}
l \\
i
\end{array}\right)(1-B E R(d))^{l-i} B E R^{i}(d)
$$

Since we use erasure code, we suppose we can reconstruct $k$ original data packets by receiving any $k$ packets out of $k+s$ packets, with $s$ check packets. Therefore, the probability of success is modified as follows:

$$
\begin{aligned}
p_{E C}\left(k, s, p_{D}, p_{C}\right)= & \sum_{i=0}^{k} \sum_{j=k-i, j \leq s}^{s}\left(\begin{array}{l}
k \\
i
\end{array}\right) p_{D}^{i}\left(1-p_{D}\right)^{k-i} \\
& \left(\begin{array}{c}
s \\
j
\end{array}\right) p_{C}^{j}\left(1-p_{C}\right)^{s-j}
\end{aligned}
$$

where $p_{D}$ and $p_{C}$ are the probabilities of success to transmit one data and check packet over one hop respectively.

As we are interested in the reliability performance of each scheme, we define the probabilities of success $p_{1}, p_{2}, p_{3}$ and $p_{4}$ for Scheme 1, 2, 3 and 4 of $k$ data packets over one hop by:

$$
\begin{aligned}
p_{1} & =p_{B C H}^{k}(l, t, d) \\
p_{i} & =p_{E C}\left(k, s, p_{D}, p_{C}\right)
\end{aligned}
$$

for $i=\{2,3,4\}$ and with the followings $p_{D}$ and $p_{C}$ :

Scheme 2

$$
p_{D}=p_{C}=p(l-\phi, d)
$$

Scheme 3

$$
\begin{aligned}
& p_{D}=p_{B C H}(l, t, d) \\
& p_{C}=p(l-\phi, d)
\end{aligned}
$$

Scheme 4

$$
p_{D}=p_{C}=p_{B C H}(l, t, d)
$$

where $\phi$ is the overhead due to $\mathrm{BCH}$ encoding.

Figures 4(a) and 4(b) show the difference between the four schemes in term of probability $p_{i}$ for 1 and 10 hops. In these figures, the depth is $100 \mathrm{~m}$ and we use $\mathrm{BCH}$ with $m=10$ and $t=2$. Therefore, the packet length is $l=1023$ and the overhead $\phi=20$. The source sends 4 data packets and 3 check packets with a source level $S L$ of $122 \mathrm{~dB}$. We consider a scheme to be reliable if it achieves a probability equal or greater than $90 \%$ (horizontal dashed line). We clearly see that Scheme 2 is the poorest while Scheme 4 is the best. For instance with 10 hops, Scheme 2 is already not reliable at a distance of $25 \mathrm{~km}$ while Scheme 4 remains around $100 \%$ up to $50 \mathrm{~km}$.

\section{B. Energy}

For commercial hydrophones [13], the energy needed to receive a packet is typically around one fifth of the transmitted energy. In addition, we assume that if the frequency is $f^{*}(d)$ $\mathrm{kHz}$, the available bit rate is $r=f^{*}(d) \mathrm{kb} / \mathrm{s}$. Therefore, the energies to transmit $E_{t}$ and receive $E_{r}$ a $l$ bits packet are

$$
\begin{aligned}
& E_{t}(l)=P_{t} \frac{l}{r} 10^{-3} \\
& E_{r}(l)=\frac{1}{5} E_{t}(l)
\end{aligned}
$$

Since a packet could be encoded with $\mathrm{BCH}$, we should look at the energy requirements of the encoding and decoding processes. The encoding process uses a linear-feedback shift register and thus the encoding energy is negligible. On the other hand, the decoding energy for a packet of length $l$ is given by

$$
E_{\text {dec }}(l, t)=\left(2 l t+2 t^{2}\right)\left(E_{a d d}+E_{m u l}\right)
$$

where $E_{m u l}$ and $E_{a d d}$ are the energy of multiplication and addition respectively in the Galois field $G F\left(2^{m}\right)$ with $m=$ $\left\lfloor\log _{2} n+1\right\rfloor$ used in BCH [14].

Erasure coding is a very powerful technique but, unfortunately, encoding and decoding processes rely on heavy operations such as vector arithmetic and matrix inversions. For small block size, [15] suggests to use finite fields operations and lookup tables. In this paper, we apply a typical small block size (4 data packets and 3 check packets). Therefore, the energy for encoding and decoding could be discarded against the energy for sending and receiving the redundancy check packets. 


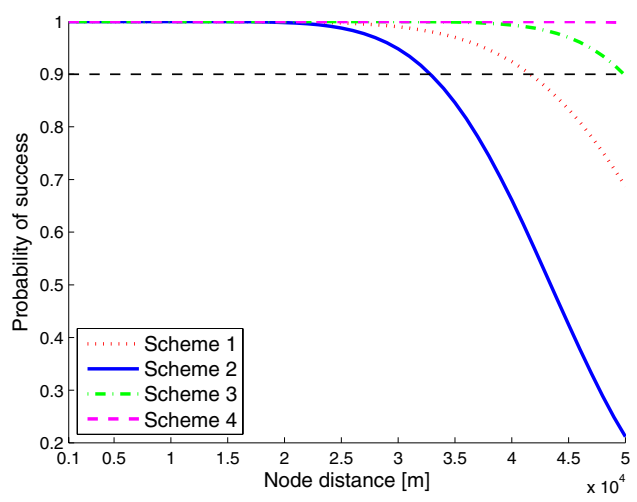

(a) 1 hop

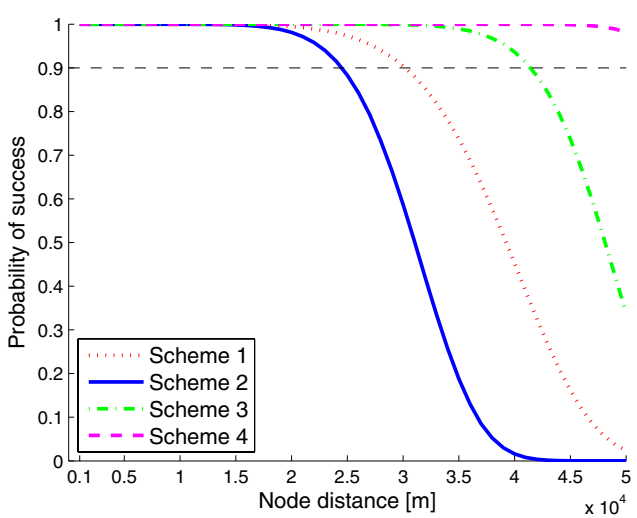

(b) 10 hops

Fig. 4. Probabilities for the non-cooperative scenario

From the preceding, the energy consumption of each scheme for $k$ data packets over one hop is

$$
\begin{aligned}
E_{1}= & k\left(E_{d e c}(l, t)+E_{t}(l)+E_{r}(l)\right) \\
E_{2}= & (k+s)\left(E_{t}(l)+E_{r}(l)\right) \\
E_{3}= & k\left(E_{d e c}(l, t)+E_{t}(l)+E_{r}(l)\right) \\
& +s\left(E_{t}(l-\phi)+E_{r}(l-\phi)\right) \\
E_{4}= & (k+s)\left(E_{\text {dec }}(l, t)+E_{t}(l)+E_{r}(l)\right)
\end{aligned}
$$

The overall expected energy consumption of Scheme $i$ for $k$ data packets over $n$ hops is given by

$$
\begin{aligned}
E_{i, t o t}(n) & =n E_{i} p_{i}^{n}+\sum_{j=0}^{n-1}(j+1) E_{i} p_{i}^{j}\left(1-p_{i}\right) \\
& =E_{i} \frac{1-p_{i}^{n}}{1-p_{i}}
\end{aligned}
$$

With $E_{a d d}=3.3 \cdot 10^{-14} \mathrm{~m}$ and $E_{m u l}=3.7 \cdot 10^{-14} \mathrm{~m}^{3}$ [14], we plot Equation 23 in Figures 5(a) and 5(b) respectively. We consider the same settings as in Figure 4. The vertical dashed lines represent the distance when a scheme has its probability below the threshold of $90 \%$.

For one hop, we should use Scheme 1 up to the second vertical dashed line, i.e. a distance of $41.59 \mathrm{~km}$, and then Scheme 3 because its energy is slightly below Scheme 4. Note that Scheme 2 can not be used because its energy is too high and its probability is below the threshold since the first vertical dashed line $(32.83 \mathrm{~km})$.

For 10 hops, the pattern is almost the same. Scheme 1 should be applied up to the second vertical dashed line $(30.17 \mathrm{~km})$. Scheme 3 is used between the second and the third vertical line $(41.43 \mathrm{~km})$, and then Scheme 4 is the best. Scheme 2 is below the probability threshold at $24.49 \mathrm{~km}$ and can not be applied for the same reasons as in one hop.

\section{COOPERATION}

We will now study the case of cooperation between nodes with the scenario depicted in Figure 6. In this scenario, the

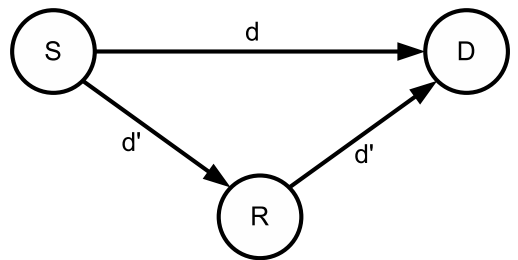

Fig. 6. A simple scenario where node $S$ wants to send packets to node $D$ with the help of the relaying node $R$.

source node $S$ wants to send packets to the destination node $D$. $S$ broadcasts packets to both Node $D$ and $R$. The relay node $R$ forwards the packets to $D$ and therefore, provides redundancy.

Without any forward correction code, we distinguish four cases for a successful transmission of a packet of length $l$. The following two equations give the total probability of success and the expected energy consumption.

$$
\begin{aligned}
p_{s}\left(l, d, d^{\prime}\right)= & p(l, d)+(1-p(l, d)) p^{2}\left(l, d^{\prime}\right) \\
E_{s}\left(l, d, d^{\prime}\right)= & \left(E_{t}(l)+2 E_{r}(l)\right) p(l, d)\left(1-p\left(l, d^{\prime}\right)\right) \\
& +\left(2 E_{t}(l)+3 E_{r}(l)\right) \\
& \left(p(l, d) p\left(l, d^{\prime}\right)+(1-p(l, d)) p^{2}\left(l, d^{\prime}\right)\right)
\end{aligned}
$$

On the other hand, there are only two cases for a failure and the total probability of failure is

$$
p_{f}\left(l, d, d^{\prime}\right)=(1-p(l, d))\left(1-p^{2}\left(l, d^{\prime}\right)\right)
$$

The expected energy consumption for a failed transmission is given by

$$
\begin{aligned}
E_{f}\left(l, d, d^{\prime}\right)= & \left(E_{t}(l)+2 E_{r}(l)\right)(1-p(l, d))\left(1-p\left(l, d^{\prime}\right)\right) \\
& +\left(2 E_{t}(l)+3 E_{r}(l)\right) \\
& (1-p(l, d)) p\left(l, d^{\prime}\right)\left(1-p\left(l, d^{\prime}\right)\right)
\end{aligned}
$$

When we only use $\mathrm{BCH}$, the probability of success and failure for one data packet is modified as follows:

$$
\begin{aligned}
p_{s, B C H}\left(l, t, d, d^{\prime}\right)= & p_{B C H}(l, t, d) \\
& +\left(1-p_{B C H}(l, t, d)\right) p_{B C H}^{2}\left(l, t, d^{\prime}\right)
\end{aligned}
$$




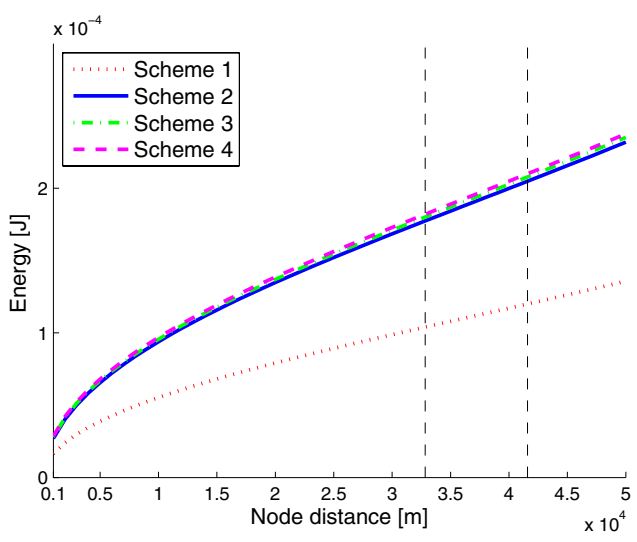

(a) 1 hop

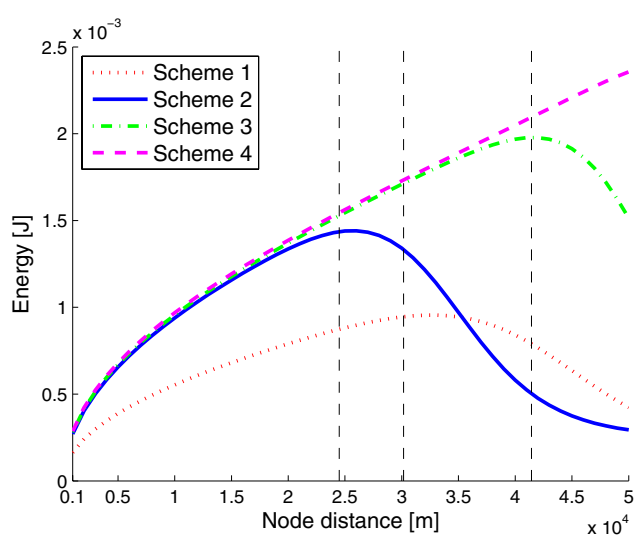

(b) 10 hops

Fig. 5. Expected energy consumptions for the non-cooperative scenario

$p_{f, B C H}\left(l, t, d, d^{\prime}\right)=\left(1-p_{B C H}(l, t, d)\right)\left(1-p_{B C H}^{2}\left(l, t, d^{\prime}\right)\right)$

and for the expected energy consumption of success and failure:

$$
\begin{aligned}
E_{s, B C H}\left(l, t, d, d^{\prime}\right)= & \left(E_{t}(l)+2 E_{r}(l)+2 E_{d e c}(l, t)\right)(30) \\
& p_{B C H}(l, t, d)\left(1-p_{B C H}\left(l, t, d^{\prime}\right)\right) \\
& +\left(2 E_{t}(l)+3 E_{r}(l)+3 E_{d e c}(l, t)\right) \\
& \left(p_{B C H}(l, t, d) p_{B C H}\left(l, t, d^{\prime}\right)\right. \\
& \left.+\left(1-p_{B C H}(l, t, d)\right) p_{B C H}^{2}\left(l, t, d^{\prime}\right)\right)
\end{aligned}
$$

$$
\begin{aligned}
E_{f, B C H}\left(l, t, d, d^{\prime}\right)= & \left(E_{t}(l)+2 E_{r}(l)+2 E_{d e c}(l, t)\right) \\
& \left(1-p_{B C H}(l, t, d)\right) \\
& \left(1-p_{B C H}\left(l, t, d^{\prime}\right)\right) \\
& +\left(2 E_{t}(l)+3 E_{r}(l)+3 E_{d e c}(l, t)\right) \\
& \left(1-p_{B C H}(l, t, d)\right) p_{B C H}\left(l, t, d^{\prime}\right) \\
& \left(1-p_{B C H}\left(l, t, d^{\prime}\right)\right)
\end{aligned}
$$

with $p_{B C H}$ defined in Equation 12.

With blocks of $k$ packets, we have for Scheme 1 the probability and energy of success and failure:

$$
\begin{aligned}
p_{s, 1}= & p_{s, B C H}^{k}\left(l, t, d, d^{\prime}\right) \\
p_{f, 1}= & p_{f, B C H}^{k}\left(l, t, d, d^{\prime}\right) \\
E_{s, 1}= & k E_{s, B C H}\left(l, t, d, d^{\prime}\right) \\
E_{f, 1}= & \sum_{i=0}^{k-1}\left(i E_{s, B C H}\left(l, t, d, d^{\prime}\right)\right. \\
& \left.+(k-i) E_{f, B C H}\left(l, t, d, d^{\prime}\right)\right) p_{s, 1}^{i}\left(1-p_{s, 1}\right)^{(k-i)}
\end{aligned}
$$

Schemes $i=\{2,3,4\}$ with erasure coding use Equation 13 for the probability of success $p_{s, i}$ :

$$
p_{s, i}=p_{E C}\left(k, s, p_{D}, p_{C}\right)
$$

The parameters $p_{D}$ and $p_{C}$ will be defined later. The probability of failure is the following:

$$
\begin{aligned}
p_{f, i}= & \sum_{i=0}^{k-1} \sum_{j=0}^{\min \{k-i-1, s\}}\left(\begin{array}{l}
k \\
i
\end{array}\right)\left(\begin{array}{l}
s \\
j
\end{array}\right) \\
& p_{D}^{i}\left(1-p_{D}\right)^{k-i} p_{C}^{j}\left(1-p_{C}\right)^{s-j}
\end{aligned}
$$

The next two equations describe the expected energy consumptions $E_{s, i}$ and $E_{f, i}$ for a successful and failed transmission respectively.

$$
\begin{aligned}
E_{s, i}= & \sum_{i=0}^{k} \sum_{j=k-i, j \leq s}^{s}\left(i E_{s, D}+(k-i) E_{f, D}\right. \\
& \left.+j E_{s, C}+(s-j) E_{f, C}\right) \\
& \left(\begin{array}{c}
k \\
i
\end{array}\right) p_{D}^{i}\left(1-p_{D}\right)^{k-i}\left(\begin{array}{c}
s \\
j
\end{array}\right) p_{C}^{j}\left(1-p_{C}\right)^{s-j}
\end{aligned}
$$

$$
\begin{aligned}
E_{f, i}= & \sum_{i=0}^{k-1} \sum_{j=0}^{\min \{k-i-1, s\}}\left(i E_{s, D}+(k-i) E_{f, D}\right. \\
& \left.+j E_{s, C}+(s-j) E_{f, C}\right) \\
& \left(\begin{array}{c}
k \\
i
\end{array}\right) p_{D}^{i}\left(1-p_{D}\right)^{k-i}\left(\begin{array}{c}
s \\
j
\end{array}\right) p_{C}^{j}\left(1-p_{C}\right)^{s-j}
\end{aligned}
$$

$p_{D}, p_{C}, E_{s, D}, E_{f, D}, E_{s, C}$ and $E_{f, C}$ used in the probability of a successful transmission and in Equations 37, 38 and 39 are the followings:

Scheme 2

$$
\begin{aligned}
p_{D} & =p_{C}=p_{s}\left(l-\phi, d, d^{\prime}\right) \\
E_{s, D} & =E_{s, C}=E_{s}\left(l-\phi, d, d^{\prime}\right) \\
E_{f, D} & =E_{f, C}=E_{f}\left(l-\phi, d, d^{\prime}\right)
\end{aligned}
$$


(a) 1 hop

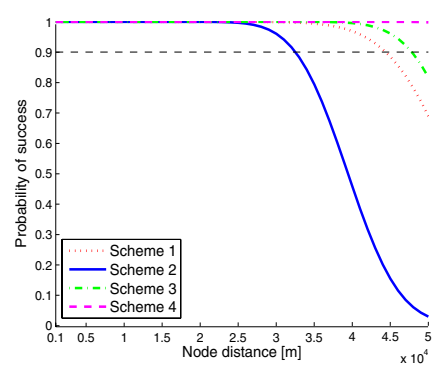

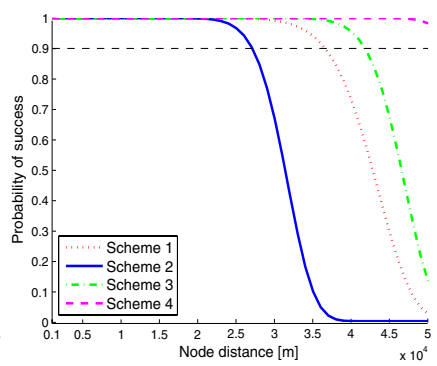

(b) 10 hops
Fig. 7. Probabilities for the cooperative scenario

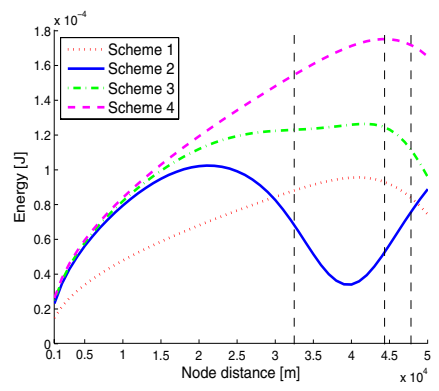

(a) 1 hop

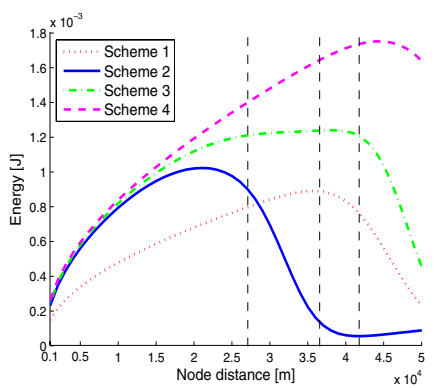

(b) 10 hops
Fig. 8. Expected energy consumptions for the cooperative scenario

Scheme 3

$$
\begin{aligned}
p_{D} & =p_{s, B C H}\left(l, t, d, d^{\prime}\right) \\
p_{C} & =p\left(l-\phi, d, d^{\prime}\right) \\
E_{s, D} & =E_{s, B C H}\left(l, t, d, d^{\prime}\right) \\
E_{f, D} & =E_{f, B C H}\left(l, t, d, d^{\prime}\right) \\
E_{s, C} & =E_{s}\left(l-\phi, t, d, d^{\prime}\right) \\
E_{f, C} & =E_{f}\left(l-\phi, t, d, d^{\prime}\right)
\end{aligned}
$$

Scheme 4

$$
\begin{aligned}
p_{D} & =p_{C}=p_{s, B C H}\left(l, t, d, d^{\prime}\right) \\
E_{s, D} & =E_{s, C}=E_{s, B C H}\left(l, t, d, d^{\prime}\right) \\
E_{f, D} & =E_{f, C}=E_{f, B C H}\left(l, t, d, d^{\prime}\right)
\end{aligned}
$$

If we replicate Figure $6 n$ times, the total expected energy consumption becomes

$$
E_{i, t o t}=n E_{s, i} p_{i}^{n}+\sum_{j=0}^{n-1}\left(j E_{s, i}+E_{f, i}\right) p_{i}^{j}\left(1-p_{i}\right)
$$

for each Scheme $i=\{1,2,3,4\}$.

Equations 36 and 40 are shown for each scheme in Figures 7 and 8 respectively for 1 and 10 hops. The parameters are

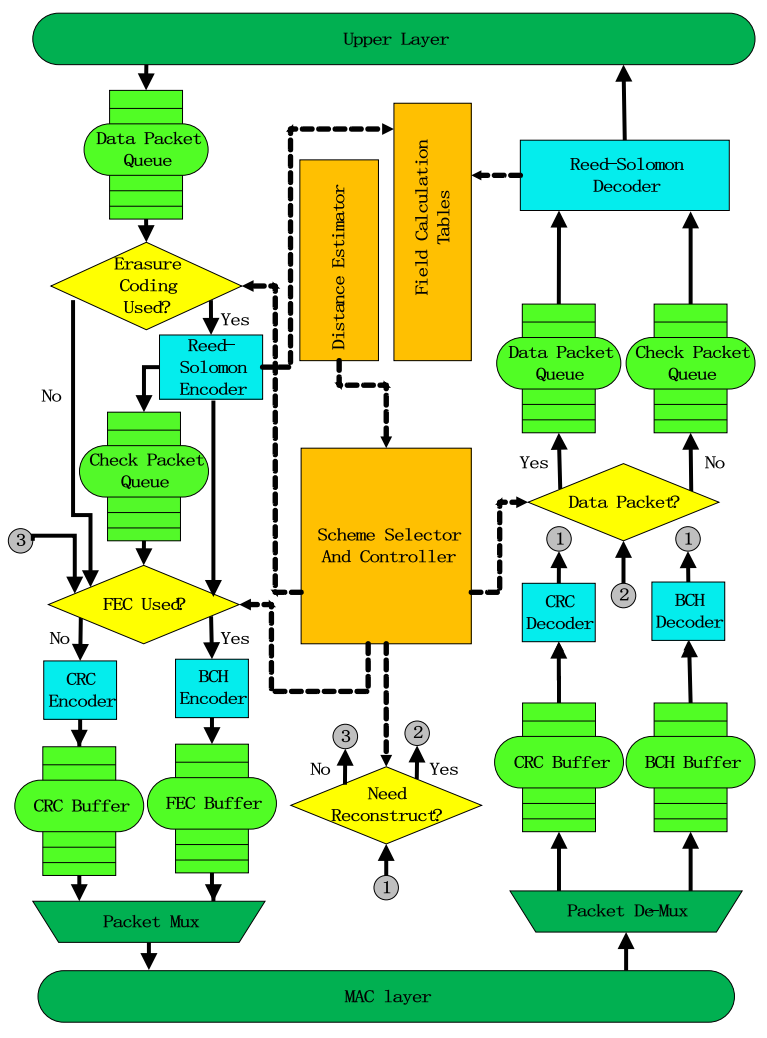

Fig. 9. ADELIN architecture

the same as in the non-cooperative scenario except that the source level $S L$ is lower, i.e. $118 \mathrm{~dB}$, and we take $d^{\prime}=\frac{d}{\sqrt{2}}$.

For one hop, Scheme 1 is used up to $29.76 \mathrm{~km}$, which is the intersection between Scheme 1 and 2. After that point, Scheme 2 has a lower energy consumption and is applied to the first vertical dashed line at $32.48 \mathrm{~km}$. Scheme 3 is the best between the first and third line $(47.8 \mathrm{~km})$. Finally, Scheme 4 should be used. Scheme 1 is below $90 \%$ at $44.34 \mathrm{~km}$.

Moreover, the strategy for 10 hops is the same for the two scenarios: Scheme 1 to the second vertical dashed line $(36.54 \mathrm{~km})$, Scheme 3 to the third line at $41.75 \mathrm{~km}$ and then Scheme 4 . The first vertical dashed line is at $27.06 \mathrm{~km}$.

\section{ADELIN}

In Sections IV and V, we discussed the relationship between the node distance and the energy efficiency and probability of each scheme. With this in mind, we propose ADELIN, an ADaptive rELIable traNsport protocol that achieves the best tradeoff between reliability and energy consumption.

The protocol is rather simple. For different distances, ADELIN uses different schemes. We define two distance thresholds $\theta_{1}$ and $\theta_{2}, \theta_{1}<\theta_{2}$. For distances shorter than $\theta_{1}$, ADELIN will use Scheme 1. Between $\theta_{1}$ and $\theta_{2}$, we have seen that Scheme 3 is the best. Above $\theta_{2}$, ADELIN applies Scheme 4 . We ignore Scheme 2 because the tradeoff between the reliability and energy consumption is too low. Figure 9 describes its architecture implemented in the simulations of the next Section. 


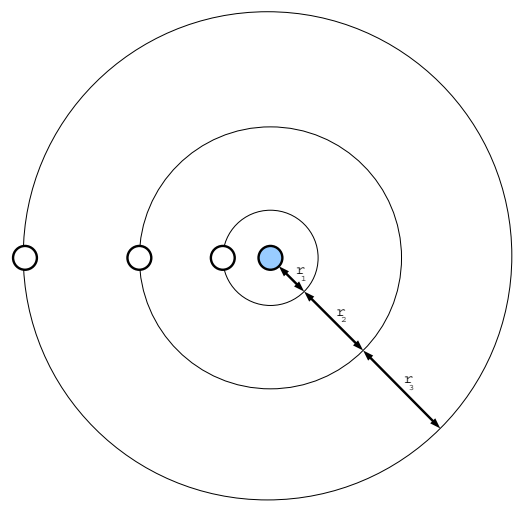

Fig. 11. Topology for one hop per ring with $r_{1}<r_{2}<r_{3}$

When a packet needs to be sent, the Scheme Selector and Controller first chooses which scheme to use according to the distance to the downstream node. The distance is estimated by either an hardware component or with approximately predefined coordinates before the deployment of the network. Once a block of data packets has reached the data packet queue, it is fed into the Reed-Solomon encoder or not depending on the chosen scheme. Based on the scheme's type, each data or check packet is placed in its respective buffer. Then they will go into the CRC or FEC encoder. Finally they are passed to the MAC layer.

When packets are received, they are first demultiplexed according to the information contained in the header and fed into the CRC or FEC buffers. After the decoding operations, packets with errors are dropped. Except for Scheme 1, packets are always reconstructed, pushed into the data or check packet queues and decoded by the Reed-Solomon decoder. The decision to reconstruct or not the packets before forwarding them could be discussed in future works. Finally, Packets are left to the upper layer.

\section{SimUlation}

To validate our analysis, we implemented the four schemes for both scenarios of Sections IV and V. We ran 50 simulations and took the average. We observe in Figure 10 that they confirm our theoretical model.

According to the observations made in Sections IV and $\mathrm{V}$, we fix the parameters of ADELIN $\theta_{1}$ and $\theta_{2}$ to $35 \mathrm{~km}$ and $42 \mathrm{~km}$ respectively. Therefore, for communication ranges shorter than $35 \mathrm{~km}$, ADELIN uses Scheme 1. Between 35 and $42 \mathrm{~km}$, Scheme 3 is preferred. Above $42 \mathrm{~km}$, ADELIN employs Scheme 4.

To compare our protocol, we use the topology depicted in Figure 11, where $r_{1}<r_{2}<r_{3}$ is the distance between each node. In our case, we choose $r_{1}=20 \mathrm{~km}, r_{2}=40 \mathrm{~km}$ and $r_{3}=50 \mathrm{~km}$. The central node is the sink and communications go from the outer ring to the sink. We vary the number of hops inside each ring from 1 to 3 hops. In one ring, all hops have the same distance between them. Note that for the cooperative scenario, we consider one hop as the whole Figure 6.
Again, we keep the same settings than in the previous simulations and we clearly see in Figure 12 that ADELIN performs better than the three other schemes. It achieves a better tradeoff between the probability of success and the energy consumption for the two scenarios with single and multihop communications. Although the cooperative scenario uses a lower source level and thus consumes less energy, the reliability remains higher than the non-cooperative one. Therefore, the broadcast property of acoustic channels helps us to save energy.

\section{CONCLUSION}

Due to the singular features of underwater environments, feedback-based protocols like automatic repeat request (ARQ) mechanisms are inconvenient. Therefore, we have naturally focused our work on redundancy solutions.

We have studied four schemes that combine forward error correction mechanisms at the bit and/or packet level to increase the reliability. Thanks to the broadcast property of the environment, we have reduced the energy consumption and increased the reliability by using a cooperative approach. The numerical results indicated us to design an adaptive reliable transport protocol called ADELIN. The simulation showed that this new protocol succeeds in a better tradeoff between reliability and energy consumption.

In future works, we would like to study further the benefits of cooperation in underwater sensor networks with, perhaps, the help of game theory. The assumption to reconstruct packets at each node could be relaxed. Furthermore, we may discuss and compare other bit and packet level FECs. We could also study the effect of their parameters.

\section{ACKNOWLEDGMENT}

This work is supported in part by the National High-Tech Research and Development Plan of China (863) under Grant No. 2006AA01Z225, 2006AA09Z117 and 2006AA01Z223; the National Grand Fundamental Research Program of China (973) under Grant No. 2006CB303000; the National Natural Science Foundation of China (NSFC) under Grant No. 60573122 and 60773138.

\section{REFERENCES}

[1] I. F. Akyildiz, D. Pompili, and T. Melodia, "Challenges for efficient communication in underwater acoustic sensor networks," SIGBED Rev., vol. 1, no. 2, pp. 3-8, 2004.

[2] J. Partan, J. Kurose, and B. N. Levine, "A survey of practical issues in underwater networks," in WUWNet '06: Proceedings of the 1st ACM international workshop on Underwater networks. New York, NY, USA: ACM, 2006, pp. 17-24.

[3] M. Stojanovic, "On the relationship between capacity and distance in an underwater acoustic communication channel," in WUWNet '06: Proceedings of the 1st ACM international workshop on Underwater networks. New York, NY, USA: ACM Press, 2006, pp. 41-47.

[4] C.-Y. Wan, A. T. Campbell, and L. Krishnamurthy, "Psfq: a reliable transport protocol for wireless sensor networks," in WSNA '02: Proceedings of the 1st ACM international workshop on Wireless sensor networks and applications, 2002, pp. 1-11.

[5] F. Stann and J. Heidemann, "Rmst: Reliable data transport in sensor networks," in Proceedings of the First International Workshop on Sensor Net Protocols and Applications, 2003, pp. 102-112. 


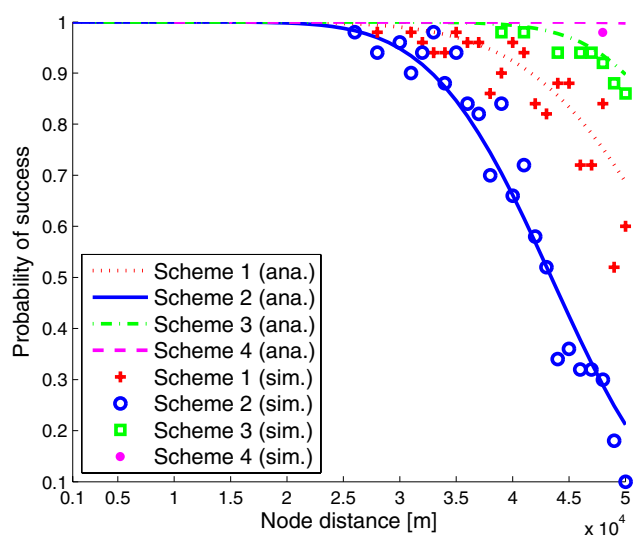

(a) Non-cooperative scenario

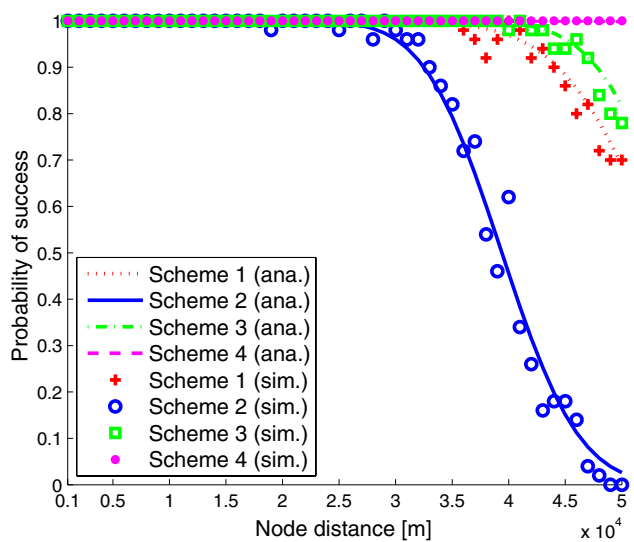

(b) Cooperatiive scenario

Fig. 10. Theoretical and simulated probabilities of success

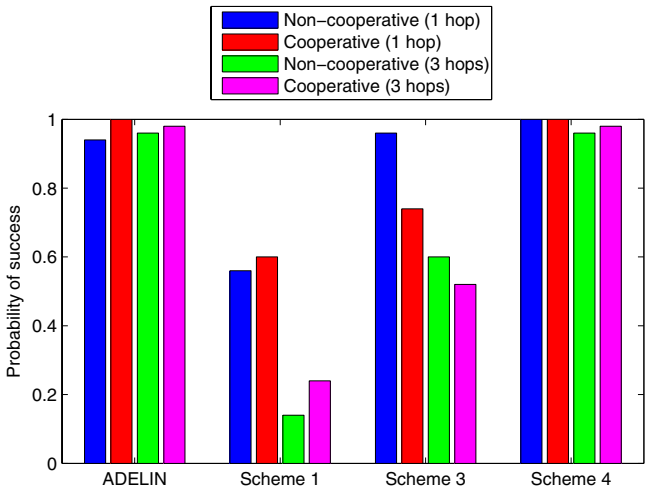

(a) Probabilities

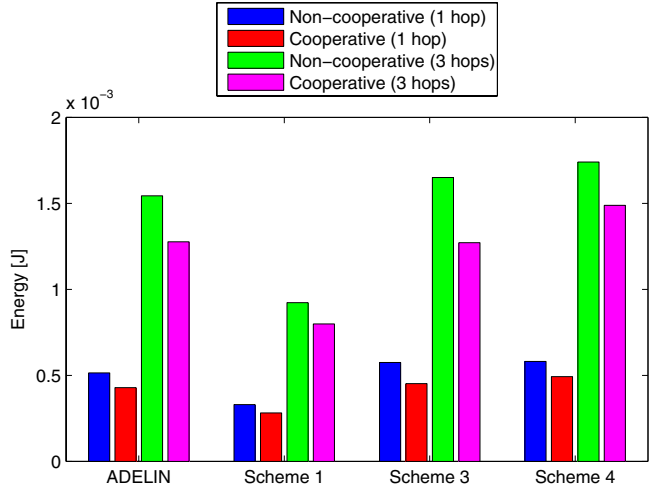

(b) Energies

Fig. 12. Comparison of probabilities and energies

[6] H.-P. Tan, W. K. G. Seah, and L. Doyle, "A multi-hop arq protocol for underwater acoustic networks," OCEANS 2007 - Europe, pp. 1-6, 18-21 June 2007.

[7] P. Xie and J.-H. Cui, "An fec-based reliable data transport protocol for underwater sensor networks," Computer Communications and Networks, 2007. ICCCN 2007. Proceedings of 16th International Conference on, pp. 747-753, 13-16 Aug. 2007.

[8] H. Medwin and colleagues, Sounds in the sea: from ocean acoustics to acoustical oceanography. Cambridge University Press, 2005.

[9] A. Goldsmith, Wireless Communications. Cambridge University Press, 2005.

[10] R. J. Urick, Principles of underwater sound, 3rd ed. McGraw-Hill, 1983.

[11] W. H. Thorp, "Analytic description of the low-frequency attenuation coefficient," Journal of Acoustical Society of America, vol. 42, no. 1, p. 270, July 1967.

[12] R. F. Coates, Underwater Acoustic Systems. Wiley, 1989.

[13] (2007) Linkquest inc. - underwater acoustic modem. [Online]. Available: http://www.link-quest.com

[14] Y. Sankarasubramaniam, I. F. Akyildiz, and S. W. McLaughlin, "Energy efficiency based packet size optimization in wireless sensor networks," Sensor Network Protocols and Applications, 2003. Proceedings of the First IEEE. 2003 IEEE International Workshop on, pp. 1-8, 2003.

[15] K. Sukun, R. Fonseca, and D. Culler, "Reliable transfer on wireless sensor networks," Sensor and Ad Hoc Communications and Networks, 2004. IEEE SECON 2004. 2004 First Annual IEEE Communications Society Conference on, pp. 449-459, 4-7 Oct. 2004. 